\title{
Some inflammatory markers and chest computerized tomography in patients with severe acute respiratory syndrome coronavirus infection
}

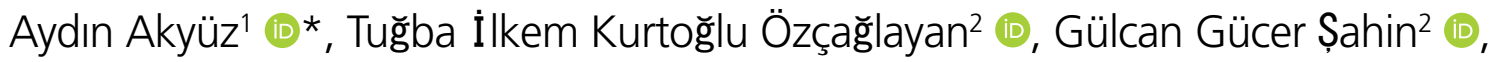 \\ Muhammet Mucip Efe ${ }^{\top} \odot$, Demet Özkaramanlı Gür ${ }^{\oplus}$, Șeref Alpsoy ${ }^{1}$
}

\begin{abstract}
SUMMARY
OBJECTIVE: We retrospectively assessed whether there was a relationship between lung complications and some easily accessible markers to predict the presence of pulmonary consolidation in patients with coronavirus disease 2019 (COVID-19).

METHODS: According to the polymerase chain reaction and chest computerized tomography results, the study was categorized into three groups. Group 1 ( $n=87$ ) included the patients with polymerase chain reaction $(+)$, group $2(n=55)$ included the patients with polymerase chain reaction (-) and chest computerized tomography $(+)$, and group $3(n=77)$ included the patients with polymerase chain reaction (-) and chest computerized tomography (-), respectively.

RESULTS: High-sensitivity C-reactive protein and increased age were associated with higher computerized tomography (CT) scores. CONCLUSION: Increased age and C-reactive protein (CRP) may suggest pulmonary infiltration on chest CT in patients with COVID-19. KEYWORDS: Coronavirus disease 2019. X-ray computed tomography. Diagnosis. C-reactive protein.
\end{abstract}

\section{INTRODUCTION}

The novel coronavirus, severe acute respiratory syndrome coronavirus (SARS-CoV-2), had an epidemic potential in 2019 and finally spread all over the world, with its status changing to a pandemic ${ }^{1,2}$. Many patients with pneumonia face intubation with a death risk. After serial autopsy reports, the clinical and pathological effects of the disease have become clear ${ }^{3,4}$. One of the main problems was a hyperactive-immune response that propagates lung dysfunction and atheroembolic events $s^{4-5}$. For this reason, many studies have been conducted to detect real pathophysiological problems.

Infections and inflammatory events cause some systemic reactants to be released, like CRP, procalcitonin, transferrin, into the body within hours or days. Since patients with COVID-19 pneumonia have a secondary infection, especially after ventilatory use, the possibility of lung disease should be considered ${ }^{6}$. These acute-phase reactants can be further increased in case of secondary infection.

$\mathrm{Hu}$ et al. have firstly described a new marker called systemic serum inflammatory index (SII) for prognosis prediction in hepatocellular carcinoma ${ }^{7}$. Subsequently, especially in cancers, other studies about the superiority of this index superiority compared to other indices, such as neutrophil-lymphocyte ratio, (NLR) were published ${ }^{8,9}$. However, a heated debate broke out about which index was the most appropriate. Although the systemic inflammatory burden of COVID-19 in patients

\footnotetext{
${ }^{1}$ Namık Kemal University, Faculty of Medicine, Cardiology Department - Tekirdağ, Turkey.

${ }^{2}$ Namık Kemal University, Faculty of Medicine, Radiology Department - Tekirdă̆, Turkey.

*Corresponding author: ayakyuzq5@gmail.com

Conflicts of interest: the authors declare there are no conflicts of interest. Funding: none.

Received on December 13, 2020. Accepted on February 07, 2021.
} 
with pneumonia is well-known, it has not been investigated in the literature as to whether NLR and SII correlate with lung involvement levels, and other clinical and laboratory parameters in patients with COVID-19 infection.

The common problem associated with these markers is whether they are specific or always correlate with disease severity ${ }^{10-12}$. This study, therefore, aimed to assess whether there is an association of lung involvement severity resulted from COVID-19 with NLR, SII, and other inflammatory markers, such as high-sensitivity C-reactive protein (Hs-CRP), procalcitonin, or ferritin, which are easy to use in practice.

\section{METHODS}

\section{Study design and protocol}

After having been approved by the local Ethics committee, the study was retrospectively conducted in patients who were admitted to the emergency department and treated after a diagnosis of SARS-CoV-2 from April 2020 to August 2020. The diagnosis and treatment of COVID-19 followed the COVID-19 Management Guidelines of the National Science Commission of Turkey.

A total of 219 consecutive patients were admitted with pre-diagnosed SARS-Cov-2. All samples for real-time polymerase chain reaction (RT-PCR) assays were obtained from a nasopharyngeal swab. All patients were detected by RT-PCR samples and chest computerized tomography (CT). In the study, group 1 included 87 consecutive SARS-Cov- 2 patients who were diagnosed with RT-PCR that was done by using commercial kits (SARS-Cov-2 Double Gene RT-PCR kit, Bioksen R\&D Technologies Ltd., Istanbul, Turkey) with endorsement by the Turkish Health Minister. According to the manufacturer, the analytical sensitivity of the kit is $99.4 \%$ and its specificity is 99\%. Group 2 included 55 patients who had negative RT-PCR findings with positive chest CT. Group 3 included 77 patients who had influenza-like-illness with a negative RT-PCR and chest CT findings.

Age, gender, the presence of hypertension, hyperlipidemia, smoking, and diabetes mellitus, as well as clinical status and progression, such as fever, dyspnea, intubation, or death, were collected from hospital records. Blood count parameters and biochemical findings were noted.

\section{Chest computerized tomography evaluation}

A 16-row multidetector scanner (GE Medical Systems, Bright Speed 16) was used to scan the study population. After raw data obtained from the Sectra PACS System, images were processed to make multi-reconstructive images.

Chest CT image analysis was calculated for every patient as done previously by Ding X et al. ${ }^{13}$ Two experienced radiologists who were blinded to the patient's RT-PCR result analyzed CT findings and scores. In case of disagreement, a third radiologist made the final decision. On chest CT scans, ground-glass opacification, consolidation, and linear opacities were evaluated. Pulmonary lesions were noted according to the anatomical lobe structures of the lungs, as follows: left upper, left lower, right upper, middle, and lower.

The involvement of each lobe in terms of quartiles was assigned a 1 to 4 score, namely mean CT score can change between 0 and 20. Scores were given according to the percentage involvement of five separate lung lobes: zero point for no involvement, 1 point for $<1-24 \%$ involvement, 2 points for $25-49 \%$ involvement, 3 points for $50-74 \%$ involvement, and 4 points for $>75 \%$ involvement (Figure 1A). Fifteen randomly selected CT scans were re-examined by the same observer and a second observer to detect inter and intra-observer variability. The inter- and intra-observer correlation coefficients of CT scores were $\geq 0.95$.

\section{Statistics}

The study parameters were analyzed by predictive analytics software (SPSS Inch, Chicago, Illinois, USA). Categorical parameters were shown absolute and relative frequencies $(\mathrm{n} \%)$. For all variables, the normality test distributions were performed using the Kolmogorov-Smirnov method. While the mean \pm standard deviation was presented for normal distributions, the median (min-max) was presented for non-normal ones. A chi-squared test was used for comparing the categorical data of the three groups. If the expected number of categorical variables in any group was less than five, the Fisher p-value test was accepted. Normally distributed data were analyzed with the ANOVA test, non-normally distributed data were compared by the Kruskal Wallis test. Dunn's pairwise comparison test was used in case the Kruskal Wallis test presented any difference. The Tukey test was used for the pairwise comparison of ANOVA test results. Linear regression analyses were used to find whether there is an association between CT scores and other variables. A 2-tailed $\mathrm{p}<0.05$ was considered significant.

\section{RESULTS}

The clinical characteristics of the study population are shown in Table 1 in terms of cardiovascular risk factors, such as age, male gender, hypertension, hyperlipidemia, smoking, and diabetes 
mellitus, as well as symptoms. The laboratory data concerning patient groups are presented in Table 2 .

The percentage of age, gender, hyperlipidemia, smoking and diabetes mellitus, hemoglobin, red cell width, mean platelet volume, D dimer, eGFR, calcium, sodium, potassium, and ferritin levels were similar in these three groups (Table 1). Groups 2 and 3 had a higher hypertension rate, CT lesion presence, and higher mean $\mathrm{CT}$ scores compared to group 1. Fever, dyspnea, cough, headache, sore throat, hospitalization time, intubation, and death rates were higher in groups 1 and 2 compared to group 3 . Group 1 and 2 variables were similar in terms of symptoms, hospitalization times, mean CT scores, intubation, and death rates.

Group 2 had the highest WBC, neutrophil, N/L ratio, SII, hs-CRP, and fibrinogen levels in the study population. Compared to group 3, groups 1 and 2 had a higher N/L ratio, Hs-CRP, AST, and procalcitonin levels (for all $\mathrm{p}$ values $<0.05$ ) (Figure 1B). SII and fibrinogen levels were similar in groups 1 and 3 .

hsCRP was correlated with $\mathrm{N} / \mathrm{L}$ ratio $(\mathrm{r}=0.387, \mathrm{p}<0.001)$ and SII $(r=0.298, p<0.001) . N / L$ ratio and SII were not correlated with CT scores and ferritin levels. Both fibrinogen and procalcitonin were correlated with $\mathrm{N} / \mathrm{L}$ ratio $(r=0.278, p=0.003$ and $\mathrm{r}=0.436, \mathrm{p}<0.001)$, and SII was correlated with procalcitonin $(\mathrm{r}=0.255, \mathrm{p}=0.007)$, but not with fibrinogen levels. Mean CT scores were correlated with CRP $(r=0.331, p<0.001)$, ferritin $(\mathrm{r}=0.336, \mathrm{p}<0.001)$ and fibrinogen $(\mathrm{r}=0.280, \mathrm{p}=0.003)$ levels, but not with procalcitonin.

Univariate linear regression analysis showed mean CT scores had correlations with increased age, hs-CRP, fibrinogen, ferritin, but not with N/L ratio, SII, and procalcitonin. However, age and hs-CRP levels appeared to be the predictors in the multivariate analysis. (Table 2).

The ROC analysis showed that hs-CRP levels $\geq 4.3 \mathrm{mg} /$ $\mathrm{dL}$ had good diagnostic value to discriminate lung involvement within the study population with $80 \%$ sensitivity and $62 \%$ specificity (area under the curve (AUC) $=0.781,95 \% \mathrm{CI}$ $0.720-0.842, \mathrm{p}<0.001)$. However, ferritin and fibrinogen levels were not good for the prediction of lung involvement compared to that of hs-CRP in the ROC analysis, $\mathrm{p}=0.255$ and $\mathrm{p}=0.238$, respectively.

\section{DISCUSSION}

We retrospectively studied patients with COVID-19 and those with a suspicion of COVID-19 due to chest CT lesions, as well as those with influenza-like symptoms. Our study findings showed that despite not having had positive RT-PCR tests, patients with chest $\mathrm{CT}$ lesions had a higher inflammation response compared to COVID-19 patients. Moreover, these patients had similar symptoms and clinical events to COVID-19 patients, such as

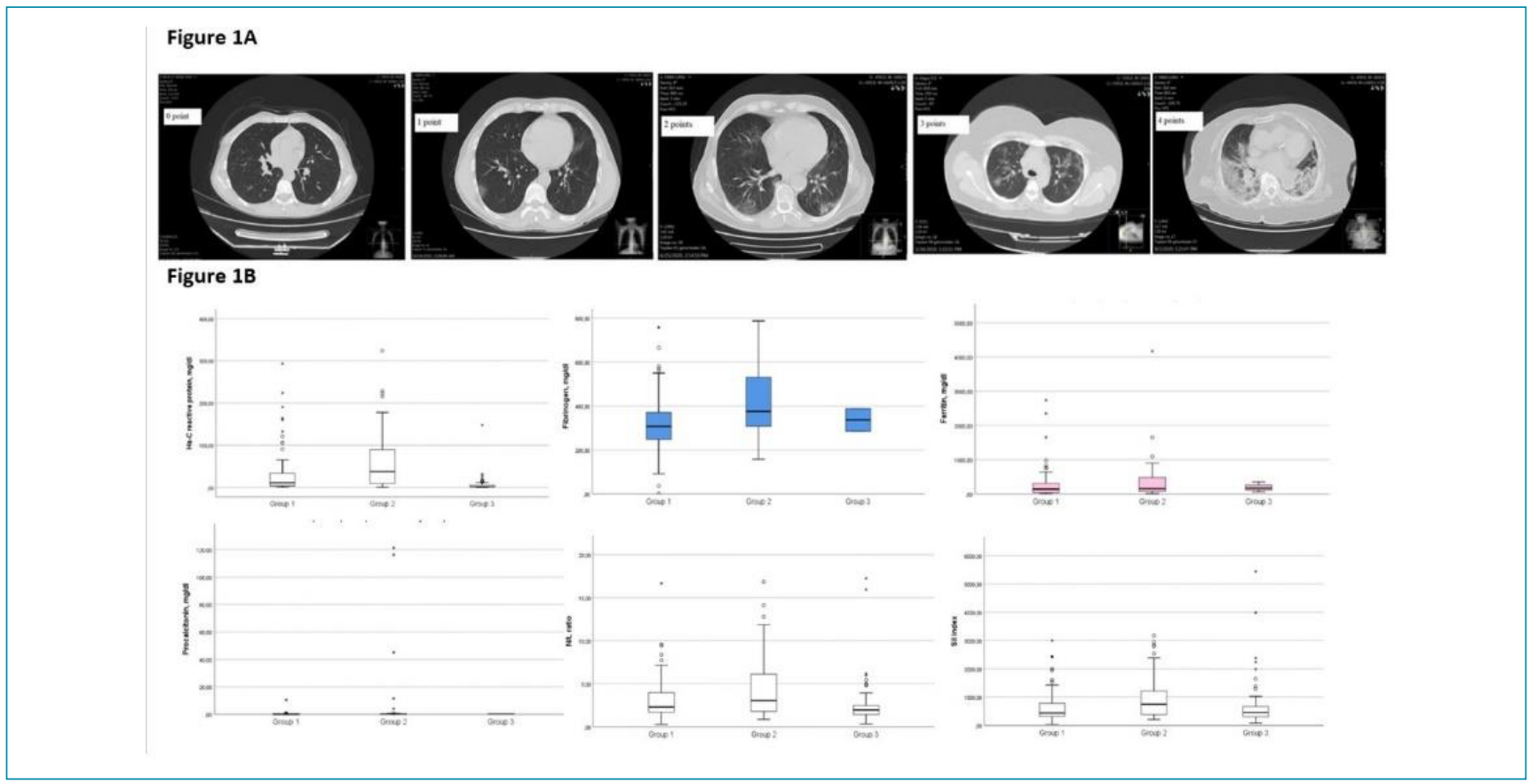

Figure 1. (A): As a result of a visual assessment of the percentage of involvement of each of the five separate lobes of the lung, scores were obtained according to the following percentages of involvement: zero point for no involvement, 1 point for $<1-24 \%$ involvement, 2 points for $25-49 \%$ involvement, 3 points for $50-74 \%$ involvement, and 4 points for $>75 \%$ involvement. (B) Inflammation markers were shown as boxplots in the three study groups. 
Table 1. Comparison of demographic, clinical characteristics, blood cell counts, and biochemical levels of laboratory data with respect to the patient groups in the study population.

\begin{tabular}{|c|c|c|c|c|}
\hline & $\begin{array}{c}\text { Group } 1 \\
n=87\end{array}$ & $\begin{array}{c}\text { Group } 2 \\
n=55\end{array}$ & $\begin{array}{c}\text { Group } 3 \\
n=77\end{array}$ & p-value \\
\hline Age, years & $49(19-84)$ & $56(20-87)$ & $52(25-86)$ & 0.081 \\
\hline Gender, male, n (\%) & $38(43.7)$ & $32(58.2)$ & $44(57.1)$ & 0.065 \\
\hline Hypertension, n (\%) & $15(17.2)$ & $22(40)$ & $24(31.2)$ & $0.037^{a, b}$ \\
\hline Hyperlipidemia, n (\%) & $8(9.1)$ & $4(7.3)$ & $10(13)$ & 0.284 \\
\hline Smoking, n (\%) & $40(46)$ & $31(56.4)$ & $24(31.2)$ & 0.052 \\
\hline Diabetes Mellitus, n (\%) & 13(14.9) & $32(21.8)$ & $8(10.4)$ & 0.384 \\
\hline Fever, n (\%) & $41(47.1)$ & $28(50.9)$ & $2(2.6)$ & $<0.001^{b, c}$ \\
\hline Dyspnea, n (\%) & $22(25.3)$ & $23(41.8)$ & $1(1.3)$ & $<0.001^{a, b, c}$ \\
\hline Cough, n (\%) & $55(63.2)$ & $31(56.4)$ & $3(0.4)$ & $<0.001^{b, c}$ \\
\hline Headache, n (\%) & $7(8)$ & $4(7.3)$ & $1(1.3)$ & $0.047^{b, c}$ \\
\hline Sore throat, $\mathrm{n}(\%)$ & 14(16.1) & $6(10.9)$ & $1(1.3)$ & $0.001^{b, c}$ \\
\hline Diarrhea, n (\%) & $4(4.6)$ & 0 & 0 & 0.077 \\
\hline Hospitalization time, day & $8.5(0-62)$ & $6(2-48)$ & $3(2-8)$ & $0.034^{b, c}$ \\
\hline Mean CT scores & $4(0-15)$ & $4(1-16)$ & 0 & $0.029^{b, c}$ \\
\hline CT lesion presence & $28(32.1)$ & $55(100)$ & 0 & $<0.001^{a, b, c}$ \\
\hline Intubation, n (\%) & $8(9.2)$ & $5(9.1)$ & 0 & $<0.001^{b, c}$ \\
\hline Death, n (\%) & $5(5.7)$ & $4(7.3)$ & 0 & $<0.001^{b, c}$ \\
\hline Hemoglobin, gr/dL & $13.3 \pm 2.1$ & $13.2 \pm 1.9$ & $13.5 \pm 1.7$ & 0.475 \\
\hline Red cell width & $14.4 \pm 1.7$ & $14.6 \pm 2.5$ & $13.9 \pm 1.9$ & 0.057 \\
\hline WBC, $\times 10^{9} / \mathrm{L}$ & $6.2 \pm 3.1$ & $9.1 \pm 4.9$ & $7.5 \pm 2.6$ & $0.001^{a, c}$ \\
\hline Neutrophils, x10\%/L & $3.3(0.1-14.2)$ & $6(1.32-17.5)$ & $3.9(1.5-11.8)$ & $0.022^{b, c}$ \\
\hline Lymphocytes, x10\%/L & $1.5 \pm 1.1$ & $1.61 \pm 1.3$ & $2.22 \pm 1.4$ & $0.007^{b, c}$ \\
\hline Platelets, x10\%/L & $209 \pm 92$ & $249 \pm 101$ & $251 \pm 78$ & $0.006^{a, b}$ \\
\hline MPV & $8.9 \pm 1.9$ & $8.6 \pm 0.8$ & $8.6 \pm 1.1$ & 0.278 \\
\hline $\mathrm{N} / \mathrm{L}$ ratio & $2.31(0.2-16.7)$ & $3.1(0.9-17)$ & $1.94(0.3-17.2)$ & $0.001^{a, b, c}$ \\
\hline SII & $437(30-3,000)$ & $750(209-3,107)$ & 465(89-5,437) & $<0.001^{a, c}$ \\
\hline $\mathrm{Hs}-\mathrm{CRP}, \mathrm{mg} / \mathrm{dL}$ & 11.1(0.5-293) & $38(0.3-323)$ & $2.3(011-147)$ & $<0.001^{a, b, c}$ \\
\hline D dimer, mg/dL & $0.55(0.2-188)$ & $0.68(0.2-21)$ & $0.62(0.19-1.27)$ & 0.228 \\
\hline eGFR, $\mathrm{mL} / \mathrm{min} / 1.73 \mathrm{~m}^{2}$ & $97 \pm 23$ & $90 \pm 25$ & $94 \pm 21$ & 0.241 \\
\hline AST, IU/L & $25(12-306)$ & $24(14-432)$ & $17.6(8-48)$ & $0.001^{a, c}$ \\
\hline ALT, IU/L & $21(0-172)$ & $24(9-432)$ & $18.5(6-48)$ & $0.047^{c}$ \\
\hline Calcium, mg/dL & $9.2 \pm 0.6$ & $9.3 \pm 0.7$ & $9.4 \pm 0.35$ & 0.850 \\
\hline Sodium, mEq/L & $138 \pm 3.7$ & $137.1 \pm 3.9$ & $139.5 \pm 2.4$ & 0.123 \\
\hline Potassium, mEq/L & $4.23 \pm 0.5$ & $4.15 \pm 0.7$ & $4.41 \pm 0.5$ & 0.121 \\
\hline Fibrinogen, mg/dL & $323 \pm 95$ & $412 \pm 156$ & $336 \pm 73$ & $0.010^{c}$ \\
\hline Procalcitonin, mg/dL & $0.61(0.2-14)$ & $0.41(0-121)$ & $0.16(0-0.20)$ & $0.012^{b, c}$ \\
\hline Ferritin, mg/dL & $139(11-2,736)$ & $157(23-4,167)$ & 170(57-350) & 0.370 \\
\hline
\end{tabular}

If p-values < 0.05 ; a: for comparing group 1 vs 2; b: for comparing group 1 vs 3 ; c: for comparing group 2 vs 3; CT: computerized tomography; AST: aspartate aminotransferase; ALT: alanine aminotransferase; eGFR: estimated glomerular filtration rate; Hs-CRP: high-sensitivity C-reactive protein; MPV: mean platelet volume; N/L: neutrophil/lymphocyte; SII: systemic serum inflammatory index; WBC: white blood cells 
Table 2. Dependent variable: CT scores of lung involvement.

\begin{tabular}{l|c|c|c} 
& $\begin{array}{c}\text { Non-standardized } \\
\text { Beta } \pm \text { SE }\end{array}$ & Adjusted R2 Model & p-value \\
\hline Age & $0.066 \pm 0.15$ & 0.091 & $<0.001$ \\
\hline Gender, male & $-0.038 \pm 0.68$ & -0.006 & 0.654 \\
\hline Hypertension & $1.611 \pm 0.77$ & 0.024 & 0.037 \\
\hline Hyperlipidemia & $1.627 \pm 1.59$ & 0.001 & 0.294 \\
\hline Smoking & $-0.113 \pm 0.69$ & -0.007 & 0.870 \\
\hline Diabetes Mellitus & $0.988 \pm 0.90$ & 0.001 & 0.275 \\
\hline N/L ratio & $0.000 \pm 0.11$ & -0.007 & 0.997 \\
\hline SII & $0.001 \pm 0.01$ & -0.047 & 0.818 \\
\hline Hs-CRP & $0.019 \pm 0.05$ & 0.086 & $<0.001$ \\
\hline D dimer & $0.011 \pm 0.02$ & -0.006 & 0.624 \\
\hline Fibrinogen & $0.009 \pm 0.03$ & 0.088 & 0.001 \\
\hline Procalcitonin & $0.008 \pm 0.02$ & -0.008 & 0.764 \\
\hline Ferritin & $0.002 \pm 0.01$ & 0.035 & 0.017 \\
\hline Multivariate & & & \\
\hline Age & $0.059 \pm 0.15$ & 0.156 & 0.002 \\
\hline HsCRP & $0.017 \pm 0.06$ & 0.156 & 0.003 \\
\hline
\end{tabular}

Hs-CRP: High-sensitivity C-reactive protein; N/L: Neutrophil/lymphocyte; SII: Systemic serum inflammatory index.

fever, cough, headache, sore throat, mean CT scores, hospitalization time, or death and intubation rates.

Since disease progression in elderly patients is mostly accelerated by an inappropriate immune response compared to young patients ${ }^{6}$, increased age was a risk factor of extensive involvement of lung lobes in the study.

CRP concentration is an extremely useful and nonspecific biochemical marker for inflammation. CRP measurement contributes significantly to organic disease screening, monitoring response to inflammation, detecting accompanying infections, and treating infection ${ }^{14}$. Compared to bacterial infections, CRP levels in viral infections are lower, but they may be high in some organ-involving viruses ${ }^{15}$. CRP is more valuable than white blood cell count in determining infection ${ }^{16-17}$. In line with earlier studies, a positive correlation between lung involvement severity in SARS-CoV-2 patients and CRP levels was not a surprise. Some authors recommend that CRP levels be used to assess the severity and outcome of patients with SARS-CoV-2 ${ }^{18-19}$. CRP values can predict the diagnosis of early severe SARS-CoV-2 infection, before CT findings ${ }^{18-19}$. Tan et al. ${ }^{11}$ found a moderate correlation of CRP with chest findings, which is in line with our results.

SII has never been evaluated in the concept of COVID19 infection to date; however, we found SII and N/L ratio are not suitable for positive CT scores. N/L ratio had a better correlation with Hs-CRP and procalcitonin than SII. Since viral infections are a common reason for lymphopenia and neutrophilia, increased N/L ratio levels are expected ${ }^{12}$. Even though we did see the highest rising values of $\mathrm{N} / \mathrm{L}$ ratio in group 2 , in which all patients had positive CT scores, no correlation with $\mathrm{N} / \mathrm{L}$ ratio was seen among the chest scores.

Surprisingly, we detected the highest fibrinogen levels in group 2. Because of higher embolic events reported, the evaluation of fibrinogen levels is particularly important in SARS$\mathrm{CoV}-2$ patients. The correlation of fibrinogen levels with N/L ratio and mean chest score may be important for anticoagulant therapy strategies in these patients, most of whom might be COVID-19 patients.

Even though they have negative PCR results, the fact that the greater mean CT scores were positively correlated with hs-CRP, ferritin, and fibrinogen levels should lead clinicians to evaluate patients with high serum levels of hs-CRP, ferritin, and fibrinogen by chest CT scanning.

There is no significant increase in procalcitonin levels in viral infections and systemic immunological diseases. Unlike cytokines and CRP, there is no significant increase in procalcitonin levels in necrosis, inflammation, and viral infections. Therefore, it is accepted that procalcitonin is specific for bacterial 
infections ${ }^{10}$. For this reason, we believe the reason for a lack of correlation between procalcitonin and lung involvement severity is a superinfection in a few patients.

As for ferritin levels correlated with lung involvement, we believe increased ferritin levels are caused by higher intracellular replication of the virus due to pneumonia. Infected host cells need iron to synthesize viral particles. As such, we believe that increased ferritin levels are a result of lung cell necrosis.

\section{Study limitations}

We did not present all data for the early, peak, and recovery stages of the disease courses. We only evaluated the clinical characteristics and laboratory data of all patients at the time of chest CT to provide clear data concerning the correlation of disease severity with lung lesions. A study showed that the greatest time of lung involvement severity during the disease was about ten days after the onset of the symptoms ${ }^{12}$. For this reason, chest CT imaging and laboratory data of our study population were obtained approximately ten days after the onset of symptoms.

\section{CONCLUSION}

The study findings made us accept that the patients who have negative RT-PCR findings with positive chest CT should be considered and treated as SARS-Cov-2 patients because they have similar CT lesions to that of COVID-19 patients. Besides, monitoring serum Hs-CRP concentrations is important for cases of suspected lung involvement in COVID-19 patients.

\section{AUTHORS' CONTRIBUTIONS}

AA: Conceptualization, Data curation, Formal analysis, Funding acquisition, Investigation, Methodology, Project Administration, Resources, Software, Supervision, Validation, Visualization, Writing-Original Draft, Writing-Review \& Editing. TIKÖ: Conceptualization, Data Curation, Formal Analysis, Funding Acquisition, Investigation, Methodology, Project Administration, Resources, Supervision, Validation, Visualization, Writing-Original Draft, Writing-Review \& Editing. GGŞ: Conceptualization, Data Curation, Formal Analysis, Funding Acquisition, Investigation, Methodology, Project Administration, Resources, Supervision, Validation, Visualization, Writing-Original Draft, Writing-Review \& Editing. MME: Conceptualization, Data Curation, Formal Analysis, Investigation, Methodology, Project Administration, Resources, Validation, Visualization, Writing-Original Draft, WritingReview \& Editing. DÖG: Conceptualization, Data Curation, Formal Analysis, Investigation, Methodology, Resources, Supervision, Validation, Visualization, Writing-Original Draft, Writing-Review \& Editing. ŞA: Conceptualization, Project Administration, Supervision, Visualization, WritingReview \& Editing.

\section{REFERENCES}

1. Shen $Y$, Zheng F, Sun D, Ling Y, Chen J, Li F, et al. Epidemiology and clinical course of COVID-19 in Shanghai, China. Emerg Microbes Infect. 2020;9(1):1537-45. https://doi.org/10.108 0/22221751.2020.1787103

2. Cheval S, Adamescu CM, Georgiadis T, Herrnegger M, Piticar A, Legates DR. Observed and potential impacts of the COVID-19 pandemic on the environment. Int J Environ Res Public Health. 2020;17(11):4140. https://doi.org/10.3390/ijerph17114140

3. Hanley B, Lucas SB, Youd E, Swift B, Osborn M. Autopsy in suspected COVID-19 cases. J Clin Pathol. 2020;73(5):239-42. https://doi.org/10.1136/jclinpath-2020-206522

4. Fox SE, Akmatbekov A, Harbert JL, Li G, Brown JQ, Heide RSV. Pulmonary and cardiac pathology in African American patients with COVID-19: an autopsy series from New Orleans. Lancet Respir Med. 2020;8(7):681-6. https://doi.org/10.1016/ S2213-2600(20)30243-5

5. Azkur AK, Akdis M, Azkur D, Sokolowska M, van de Veen $W$, Brüggen $M C$, et al. Immune response to SARS-CoV-2 and mechanisms of immunopathological changes in COVID-19. Allergy. 2020;75(7):1564-81. https://doi.org/10.1111/all.14364

6. Zhu T, Wang Y, Zhou S, Zhang N, Xia L. A comparative study of chest computed tomography features in young and older adults with corona virus disease (COVID-19). J Thorac Imaging. 2020;35(4):W97-101. https://doi.org/10.1097/ RTI.0000000000000513

7. Hu B, Yang XR, Xu Y, Sun YF, Sun C, Guo W, et al. Systemic immune-inflammation index predicts prognosis of patients after curative resection for hepatocellular carcinoma. Clin Cancer Res. 2014;20(23):6212-22. https://doi.org/10.1158/10780432.CCR-14-0442

8. Zhong JH, Huang DH, Chen ZY. Prognostic role of systemic immune-inflammation index in solid tumors: a systematic review and meta-analysis. Oncotarget. 2017;8(43):75381-8. https://doi.org/10.18632/oncotarget.18856

9. Huang $\mathrm{H}$, Liu Q, Zhu L, Zhang Y, Lu X, Wu Y, et al. Prognostic value of preoperative systemic immune-inflammation index in 
patients with cervical cancer. Sci Rep. 2019;9(1):3284. https:// doi.org/10.1038/s41598-019-39150-0

10. Chan YL, Tseng CP, Tsay PK, Chang SS, Chiu TF, Chen $J C$. Procalcitonin as a marker of bacterial infection in the emergency department: an observational study. Crit Care. 2004 Feb;8(1):R12-20. https://doi.org/10.1186/cc2396

11. Tan C, Huang Y, Shi F, Tan K, Ma Q, Chen Y, et al. C-reactive protein correlates with computed tomographic findings and predicts severe COVID-19 early. Clin Chim Acta. 2020 Jul;506:145-148. https://doi.org/10.1016/j.cca.2020.03.022

12. Li YX, Wu W, Yang T, Zhou W, Fu YM, Feng QM, et al. [Characteristics of peripheral blood leukocyte differential counts in patients with COVID-19]. Zhonghua Nei Ke Za Zhi. 2020;59(0):E003. PMID: 32114745

13. Ding $X, X u$ J, Zhou J, Long Q. Chest CT findings of COVID-19 pneumonia by duration of symptoms. Eur J Radiol. 2020;127:109009. https://doi.org/10.1016/j.ejrad.2020.109009

14. Pepys MB, Hirschfield GM. C-reactive protein: a critical update. J Clin Invest. 2003;111(12):1805-12. https://doi.org/10.1172/ $\mathrm{JCl} 18921$
15. Cermak J, Key NS, Bach RR, Balla J, Jacob HS, Vercellotti GM $C$-reactive protein induces human peripheral blood monocytes to synthesize tissue factor. Blood. 1993;82(2):513-20. PMID: 8329706

16. Çelebi S, Bulur N, Hacimustafaoglu M, Özakin C, Çakir D, Bozdemir SE, et al. Comparison of C-reactive protein, procalcitonin and serum amyloid-a levels in diagnosis of bacterial infection in children. Cocuk Enfeksiyon Dergisi. 2013;7(4):147-56. https://doi.org/10.5152/ced.2013.1562

17. Kono T, Otsuka M, Ito M, Misawa M, Hoshioka A, Suzuki M, et al. Negative C-reactive protein in children with bacterial infection. Pediatr Int. 1999;41(5):496-9. https://doi.org/10.1046/j.1442200x.1999.01126.x

18. Liu F, Li L, Xu M, Wu J, Luo D, Zhu Y, et al. Prognostic value of interleukin-6, C-reactive protein, and procalcitonin in patients with COVID-19. J Clin Virol. 2020;127:104370. https://doi. org/10.1016/j.jcv.2020.104370

19. Wang L. C-reactive protein levels in the early stage of COVID-19. Med Mal Infect. 2020;50(4):332-4. https://doi.org/10.1016/j. medmal.2020.03.007 